\title{
Interfaces
}

INTERFACES Image Texte Language

$36 \mid 2015$

Synaesthesia

\section{Émotions synesthésiques et lyrisme transgénérique}

\section{Sandra Glatigny}

\section{(2) OpenEdition}

\section{Journals}

Édition électronique

URL : http://journals.openedition.org/interfaces/237

DOI : 10.4000/interfaces.237

ISSN : 2647-6754

Éditeur :

Université de Bourgogne, Université de Paris, College of the Holy Cross

Édition imprimée

Date de publication : 1 janvier 2015

Pagination : 161-172

ISBN : 9780983175247

ISSN : $1164-6225$

\section{Référence électronique}

Sandra Glatigny, «Émotions synesthésiques et lyrisme transgénérique », Interfaces [En ligne], 36 |

2015, mis en ligne le 01 janvier 2018, consulté le 07 janvier 2021. URL : http://

journals.openedition.org/interfaces/237 ; DOI : https://doi.org/10.4000/interfaces.237

Les contenus de la revue Interfaces sont mis à disposition selon les termes de la Licence Creative Commons Attribution 4.0 International. 


\title{
ÉMOTIONS SYNESTHÉSIQUES \\ ET LYRISME TRANSGÉNÉRIQUE
}

\author{
Sandra Glatigny
}

Quand Baudelaire écrit dans «Correspondances» que les «parfums, les couleurs et les sons se répondent » (Baudelaire, 8), il ne fournit pas seulement une caution anticipée au symbolisme, il donne également l'une des clés pour repenser le lyrisme et plus largement l'émotion en littérature. En effet, dans la deuxième moitié du XIX ${ }^{\mathrm{e}}$, il faut trouver une autre voie à la transmission des sentiments et l'une de ces voies consiste à céder la place aux sensations. Dans cette perspective, la plupart des poètes lyriques recourent à une poétique que l'on pourrait qualifier de synesthésique dans la mesure où elle cherche à faire converger les différentes perceptions sensorielles. Mon hypothèse est que la fusion des sens s'opère grâce au dispositif lyrique dont le caractère transgénérique est capable de dépasser les catégories perceptuelles et artistiques : il s'agit de générer l'émotion par l'intermédiaire des sensations. Après avoir exposé les problèmes posés par l'analyse de la synesthésie en littérature, nous envisagerons les modalités de cette poétique synesthésique.

\section{Remarques préliminaires : problèmes posés par l’analyse de la synesthésie}

Il s'agit d'abord de s'interroger sur la nature de la synesthésie. Si l'on part du constat simple suivant lequel la fusion des sens nécessite de solliciter plusieurs sens à la fois, divers obstacles se présentent. D'abord, il faut que le lecteur éprouve physiquement et concrètement les sensations, les vibrations issues de l'ouie, de la vue ou du toucher. Or, ce que le lecteur projette simultanément dans la littérature, ce sont avant tout des « objets mentaux » (Bonnefoy, 214), même si sa réception du texte passe par une lecture à haute voix. En outre, la synesthésie ou plutôt la pseudo-synesthésie, suivant la classification d'Hervé-Pierre Lambert ${ }^{1}$, peut se concevoir uniquement si l'on prend en compte les valeurs culturelles attribuées aux sons, aux couleurs et aux formes, etc. Sans aller jusqu'à la théorie de l'instrumentation verbale, développée par René Ghil², les intertextes artistiques et l'intériorisation de procédés extralittéraires génèrent dans l'esprit du lecteur des représentations d'où découlent les

1 Hervé-Pierre Lambert. «La synesthésie. Vues de l'intérieur », in Épistémocritique, Volume VIII - Printemps 2011, ISSN 1913-536X. http://www.epistemocritique.org/spip.php?article210.

2 René Ghil. Traité du verbe. Paris : Giraud, 1886. 
sensations associées. À l'instar de Dawid Howes, nous distinguerons donc l'expérience physiologique et perceptuelle de l'expérience conceptuelle et intellectuelle (Howes, 39). Par exemple, lorsque le poème "Le paysage dans le cadre des portières $»^{3}$ débute, le lecteur se trouve plongé dans une expérience à dominante visuelle mais pour lui faire voir et sentir ce qui défile par la portière, Verlaine mobilise les autres sens :

Le paysage dans le cadre des portières

Court furieusement, et des plaines entières

Avec de l'eau, des blés, des arbres et du ciel

Vont s'engouffrant parmi le tourbillon cruel

Où tombent les poteaux minces du télégraphe

Dont les fils ont l'allure étrange d'un paraphe.

- Une odeur de charbon qui brûle et d'eau qui bout,

Tout le bruit que feraient mille chaînes au bout

Desquelles hurleraient mille géants qu'on fouette ;

Et tout à coup des cris prolongés de chouette. (146)

La sensation tactile de la vitesse et de l'air est suggérée phoniquement par l'énumération et le jeu des correspondances. La fréquence des fricatives [f] et des [v], à la rime dans le premier quatrain et à l'intérieur des vers, intervient dans le processus par l'intermédiaire de l'harmonie imitative. Mais peut-on dire que Verlaine fait sentir le défilement et la succession d'images ou qu'il la suggère à l'imagination du lecteur ? Dans la deuxième strophe, l'association entre l'ouïe et la vue trouve la médiation de l'imaginaire infernal. L'image naît par la redondance hyperbolique " mille chaines », " mille géants » et de la suggestion phonique. Cette strophe fait entendre un grand vacarme grâce aux allitérations associées au réseau lexical du bruit. L'enjambement entre la locution prépositionnelle et le pronom relatif participe largement à faire entendre l'image du bruit ou à faire voir le bruit parce qu'elle souligne une rime homonyme. On le voit, les perceptions résultent à la fois de l'expérience sensorielle et de la dimension culturelle et intellectuelle de l'écriture.

Ensuite, pour que la fusion des différentes perceptions sensorielles se réalise, les différents systèmes sémiotiques qui les suscitent doivent coexister. Or l'analyse se heurte à des fonctionnements hétérogènes. Tandis que la langue implique une approche majoritairement intellectuelle nécessitant de

3 Paul Verlaine. La bonne Chanson. Euvres poétiques complètes, édition Jacques Borel. Paris : Gallimard, «Bibliothèque de la Pléiade», 1962. p. 146. 
construire le sens à partir du lien qu'entretient le signe avec son référent, les systèmes iconographique et musical mobilisent d'autres processus. Tous deux génèrent un rapport plus unilatéral mais aussi plus complexe entre le signe et la réalité. Dans les arts picturaux, le signe est presque entièrement réduit à un signifiant, perçu par l'œil, et qui renvoie de manière plus ou moins univoque à la réalité. Bien évidemment, de la peinture figurative à la peinture abstraite, la distance se creuse et rappelle le fonctionnement linguistique. Dans les arts sonores, l'oreille perçoit les sons, les rythmes, l'harmonie et la mélodie par un signe qui se suffit à lui-même. Ainsi Jankélévitch a-t-il pu dire que la musique était un " espressivo inexpressif » (Jankélévitch, 25), c'est-à-dire une forme d'expression indicible, intraduisible dans le langage et dans la réalité. La difficulté réside notamment dans le fait que la littérature engage l'expérience conceptuelle avant l'expérience perceptuelle alors que c'est l'inverse pour la peinture et la musique.

Enfin, l'étude de la synesthésie est d'autant plus délicate que ces arts et ces systèmes sémiotiques paraissent incompatibles : ils font appel à des modalités d'expression, de perception et de réception

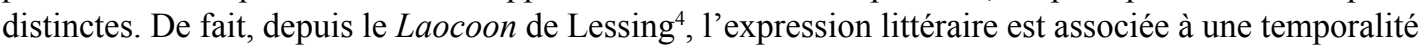
et à une linéarité qui suppose une saisie successive et analytique. En revanche, l'expression picturale appelle une saisie globale, spatialisée et synthétique. Si l'on prend l'exemple de « Voyelles », Rimbaud essaie de faire voir et entendre tout à la fois, en procédant par superposition d'images mentales. Cette écriture appelle une lecture paradigmatique quand le texte invite à une saisie linéaire et analytique, dans l'ordre syntagmatique :

A noir, E blanc, I rouge, U vert, O bleu : voyelles, Je dirai quelque jour vos naissances latentes :

A, noir corset velu des mouches éclatantes

Qui bombinent autour des puanteurs cruelles,

Golfes d'ombre ; E, candeur des vapeurs et des tentes,

Lances des glaciers fiers, rois blancs, frissons d'ombelles ;

I, pourpres, sang craché, rire des lèvres belles

Dans la colère ou les ivresses pénitentes ; (Rimbaud, 279)

On mesure la difficulté de l'analyse. L'écriture génère une succession d'images empruntées à une réalité concrète : le lecteur établit un lien direct entre les couleurs et des éléments qui lui sont familiers,

4 Gotthold Ephraim Lessing. Laocoon, ou Des limites respectives de la poésie et de la peinture (1766-1768).

Paris : Hermann, 1990. 
il fait appel à l'expérience mnémonique et sensorielle de cette réalité. Le « noir corset velu des mouches éclatantes » opère un grossissement visuel, conviant aussi le toucher. Le verbe " bombiner » mobilise la perception auditive sur les plans du signe et de son référent. Quant aux " puanteurs cruelles », le commentaire est inutile. De même, le E blanc se retrouve en tant que signifiant mais aussi en tant que signifié avec « candeur, tentes, lances, glaciers, blancs, ombelles ». La surcharge phonique et visuelle de l'évocation instaure un système d'échos entre le signifiant de la couleur attribuée à la lettre et les objets visuels, auditifs voire olfactifs qui lui sont associées. Le texte, l'image et le son, le conceptuel et le perceptuel entrent en concurrence. Tel est bien le problème que pose la synesthésie : elle brouille les perceptions et partant, les moyens de communication, propres à chaque art.

Malgré tous ces problèmes, la poétique synesthésique apparaît comme un aspect incontournable du lyrisme parce qu'elle témoigne de sa transgénéricité. Elle contribue à brouiller les termes de la communication et permet de dépasser les catégories génériques et artistiques.

\section{Quel dispositif lyrique pour cette poétique synesthésique ?}

Ce dispositif, pour reprendre un terme cher à la critique toulousaine, doit jeter un pont entre les différents pôles pour opérer une réception multisensorielle. La poétique mise en œuvre suppose une transformation des systèmes sémiotiques à l'origine des sensations. Nombreux sont les poètes qui ont tenté de modifier le fonctionnement du texte. Certains ont exploité la langue comme un simple matériau susceptible de faire voir ou de faire percevoir une vibration. Le mot n'est pas envisagé pour son signifié mais pour son signifiant. Dans Les Amours jaunes, Corbière ne cesse de déstabiliser le lecteur en employant le mot comme son ou comme image. Le lecteur est sans cesse arrêté par l'incongruité du signe, qu'il s'agisse des variations de polices, de mises en page, de ponctuations fortes. Dans « Chapelet » (Corbières, 92), il multiplie les emprunts à d'autres langues avec le latin, l'espagnol ou le flamand. Dans « Un sonnet ou la manière de s'en servir » (Corbières, 37), il introduit des signes mathématiques, inhabituels dans le texte littéraire :

- Je pose 4 et $4=8$ ! Alors je procède,

En posant 3 et $3 !-$ Tenons Pégase raide :

«Ô lyre ! Ô délire ! Ô... »- Sonnet - Attention ! (37)

L'attention est attirée sur la dimension iconique du mot. Cette utilisation du signe privilégie une appréhension synthétique et globale par opposition à une approche linéaire et analytique. Le déroulement de la chaîne syntagmatique est également modifié par la déconstruction syntaxique qui 
libère l'appréhension sensorielle. Parmi les exemples les plus significatifs, on peut citer Un coup de dés jamais n'abolira le hasard de Mallarmé (Mallarmé, 462-63) :

\section{LE MAITTRE}

surgi
inférant

de cette conflagration

comme on menace

L'unique Nombre qui ne peut pas

plutôt.

que de jouer

en maniaque chenu

la partie

au nom des flots hors d'anciens calculs

où la manouvre avec l'âge oubliée

jadis il empoignait la barre

à ses pieds

de l'horizon unanime

prépare

s'agite et mêle

au poing qui l'étreindrait

un destin et les vents

être un autre

Esprit

pour le jeter

dans la tempête

en reployer la division et passer fier

écarté du secret qu'il détient

envahit le chef

coule en barbe soumise

direct de l'homme

sans nef

n'importe

où vaine 
Sur cette page, la typographie, les blancs forment un rythme évoquant une partition comme le souligne Mallarmé dans la préface : «Ajouter que dans cet emploi à nu de la pensée, avec retraits, prolongements, fuites, ou son dessin même, résulte pour qui veut lire à haute voix, une partition » (Mallarmé, 455). L'iconicité de cette page est complexe. Le modèle de l'estampe, explicitement revendiqué par le poète (Mallarmé, 1582), vient croiser celui de la partition musicale. L'emprunt à plusieurs modes de représentations favorise les échanges et les transferts sémiotiques en brouillant les catégories de la vue et de l'audition. Le sens de lecture linéaire est mis en échec. La déconstruction spatiale et syntaxique de la métaphore marine invite à un mode de lecture paradigmatique ou synthétique.

Pour faire fusionner les différentes perceptions sensorielles, les poètes tentent de faire converger les différents systèmes sémiotiques en développant une écriture plurimédiatique. On connaît les phénomènes de transpositions artistiques largement étudiés, par Bernard Vouilloux ${ }^{5}$ ou Daniel Bergez ${ }^{6}$. Gautier, Baudelaire, Rimbaud, pour ne citer qu'eux, ont utilisé des moyens empruntés aux autres arts. Rien d'original au XIX ${ }^{\mathrm{e}}$ sauf si l'on envisage le rapport qu'entretiennent entre elles ces différentes médiations. Chez Verlaine, c'est la concurrence qui règne :

Quand Marco chantait, ses mains, sur l'ivoire,

Évoquaient souvent la profondeur noire

Des airs primitifs que nul n'a redits,

Et sa voix montait dans les paradis

De la symphonie immense des rêves,

Et l'enthousiasme alors transportait

Vers des cieux connus quiconque écoutait

Ce timbre d'argent qui vibrait sans trêves,

Quand Marco chantait. (Verlaine, « Marco », 86-87)

Malgré leur rapprochement à la rime, les termes « ivoire » et «noire » font apparaître un contraste pictural et iconique : alors que le son élabore un parallèle, l'esprit construit un objet mental antithétique à partir du référent chromatique. Alors que le chant, dans l'ordre de la fiction, renvoie à une forme

$5 \quad$ Bernard Vouilloux. La Peinture dans le texte. XVIIIe-XXe siècles. Paris : CNRS Éditions, coll. « CNRS Langages », 1995 (réimp. 1997).

6 Daniel Bergez. Littérature et peinture. Paris : A. Colin, 2004. 
d'hymne, la matérialité du signifiant vient opérer une résistance et tout particulièrement la répétition des palato-vélaires dans le vers antépénultième. Cette impression est de nouveau contrée par le bouclage strophique qui met en valeur le « chan[t] » de Marco. Dans le "Sonnet allégorique de lui-même », Mallarmé joue sur la contradiction entre l'approche textuelle et l'approche sonore : «Aboli bibelot d'inanité sonore » (Mallarmé, 68). La signification du vers évoque le vide. Pourtant, la dimension auditive, presque gustative, de la sonorité suggère la plénitude. Le fonctionnement sémiotique de chaque médiation se trouve modifié par cette tension, générant ainsi une rencontre des sens.

Cette écriture synesthésique et plurimédiatique conduit à une déconstruction du conceptuel. En cela, elle est particulièrement adaptée au lyrisme. En effet, vecteur d'un affect, abstrait, indicible, infigurable et singulier, le lyrisme s'élabore à partir de ce qui a priori s'oppose à une mise en forme et à une saisie intellectuelle. Or, pour opérer la fusion des sens, la poétique synesthésique remet en cause des objets mentaux élaborés par le jeu des analogies culturelles. Chez Rimbaud, des vers comme ceux de « Barbare » superposent les approches visuelles et tactiles ainsi que des sensations antithétiques : « Le pavillon en viande saignante sur la soie des mers et des fleurs arctiques » ou les «brasiers pleuvant aux rafales des givres » (Rimbaud, 489). L'image du monde en est totalement renouvelée, hors du champ habituel de référence. Dans « Marine », le brouillage métaphorique des éléments prend volontairement le contrepied des attentes culturelles du lecteur / spectateur :

Les chars d'argent et de cuivre

Les proues d'acier et d'argent

Battent l'écume,

Soulèvent les souches de ronces. (Rimbaud, 485)

Cette démarche rejoint celle de Verlaine qui cultive la confrontation entre les différents gestes de figuration. De même que dans " Nuit du Walpurgis classique ", "Watteau [est] rêvé par Raffet ", de même dans "Jésuitisme ", le " Rêve mi-pourri / Beugle un De profundis sur l'air du Tradéri » (Verlaine, 71 et 75). Dans « Malines », le voyage en train paraît immobile alors que défile un « Sahara de prairies, / Trèfle, luzerne et blancs gazons » (Verlaine, 201). La notation descriptive évoque une étendue dominée par les couleurs chaudes, associées à la perception de la chaleur, immédiatement contredite par la mention des prairies et des blancs gazons, suggérant la verdure et le froid. Baudelaire avait déjà largement amorcé ce mouvement en brouillant les catégories esthétiques et morales. Tout le monde se souvient de ces strophes extraites d'« Une Charogne » : 
Les mouches bourdonnaient sur ce ventre putride,

D'où sortaient de noirs bataillons

De larves, qui coulaient comme un épais liquide

Le long de ces vivants haillons.

Tout cela descendait, montait comme une vague,

Ou s'élançait en pétillant ;

On eût dit que le corps, enflé d'un souffle vague,

Vivait en se multipliant.

Et ce monde rendait une étrange musique,

Comme l'eau courante et le vent,

Ou le grain qu'un vanneur d'un mouvement rythmique

Agite et tourne dans son van. (Baudelaire, 23)

Le lecteur mobilise sa mémoire olfactive, auditive et visuelle. La perception est renforcée par l'alternance de vers longs et courts et le rejet : « de noirs bataillons / De larves », mimant le mouvement des insectes. Mais, alors que surgit l'image repoussante et dysphorique de la décomposition, elle se heurte à une nouvelle vision, celle de la vague, soutenue, elle-aussi, par la versification. L'ouïe devient l'intermédiaire qui permet d'associer vue et odorat, d'assurer le passage de l'image textuelle à l'image sensorielle.

Laforgue ou Corbière, quant à eux, introduisent des objets populaires comme la chanson et la réclame qui incitent le lecteur à repenser les valeurs et par conséquent la dimension conceptuelle de l'objet mental généré par l'écriture. Chez Laforgue, les onomatopées sont souvent associées à la chanson comme dans la « Complainte de Lord Pierrot» (Laforgues, 115), qui se réfère à «Au clair de la lune ». En l'occurrence, le chant populaire est à ce point inscrit dans l'esprit du lecteur que l'intertexte gomme le passage du conceptuel au sensoriel : il conduit à privilégier l'interprétation orale et la perception auditive.

Pour que la fusion des sens s'opère dans le texte, les poètes s'efforcent de modifier le fonctionnement du système linguistique de telle sorte qu'objets mentaux et objets perceptuels entrent en concurrence. Dès lors, la synesthésie génère une tension entre l'approche linéaire, conceptuelle et analytique et une appréhension globale, sensible et synthétique. 


\section{La transgénéricité d'un lyrisme renouvelé}

La mobilisation conjointe ou concurrentielle des différentes sensations transforme en profondeur l'expression des sentiments et leur réception. Elle témoigne du caractère transgénérique du lyrisme entendu comme principe vectoriel des émotions, qui, grâce à sa dynamique critique interne, transcende les classifications génériques et artistiques (Moncond'huy - Scepi, 9).

La poétique synesthésique génère une réception qui dépasse les catégories perceptuelle et intellectuelle, faisant de la sensation la médiation de l'expression émotionnelle. De fait, la sensation implique une appréhension, voire une préhension immédiate de la réalité alors que l'affect, l'émotionnel est médiatisé, s'inscrit dans une relation entre le sujet et le monde comme l'a montré Käte Hamburger à propos de l'énonciation lyrique (Hamburger, 220). C'est dans cette immédiateté de la relation sensorielle que la poétique synesthésique participe du renouvellement du lyrisme tant du point de vue de la création que de la réception de l'œuvre. L'expression des sentiments ne passe plus par une écriture fondée sur une harmonie conceptuelle mais sur la rencontre entre sensation et émotion. Ainsi Corbière, en contestant la relation entre médiation et perception sensorielle, déplace-t-il l'affect dans l'intervalle entre l'intelligible et le sensible, comme dans « À une demoiselle », sous-titré " pour piano et chant» :

Déchiffre au clavecin cet accord de ma lyre,

Télégraphe à musique, il pourra le traduire ;

Cris d'os, dur, sec, qui plaque et casse-plangorer...

Jamais ! - la Clef de Sol n'est pas la clef de l'âme,

La Clef de fa n'est pas la syllabe de femme,

Et deux demi-soupirs...Ce n'est pas soupirer. (Corbières, 123)

Corbière fait référence au lyrisme romantique et notamment à Lamartine en filant ironiquement la métaphore de la lyre du cœur. Du point de vue sensoriel, la répétition des palato-vélaires et les monosyllabes heurtent l'oreille. Cette espèce d'agression auditive vient contredire l'harmonie des sens et des sentiments. Dès lors, ne reste plus que le dialogue intersémiotique qui laisse voir l'amertume et l'impuissance du poète à exprimer ses émotions. Iconoclaste, l'écriture de Corbière tente de dissoudre la singularité émotionnelle dans l'universalité concrète des sensations : c'est au lecteur d'actualiser une configuration affective laissée en suspens par la concurrence des objets mentaux et des sensations.

De plus, la poétique synesthésique participe à la transgénéricité du lyrisme par sa dimension métadiscursive. Les intertextes artistiques mettent au jour la dualité du lyrisme, à la fois texte et métatexte, réflexion de l'émotion et réflexion sur l'émotion. Cette écriture insuffle une dynamique 
critique qui replace les sentiments sous le signe du mouvement et de la circulation affective. La "Complainte de l'orgue de Barbarie » renvoie à un instrument qui, comme l'a montré Laurence Tibi, symbolise le heurt ${ }^{7}$. Ce choix témoigne d'un refus des conditions habituelles de réception et des imaginaires lyriques traditionnels.

Orgue, orgue de Barbarie,

Don Quichotte, souffre-douleur,

Vidasse, vidasse ton cœur,

Ma pauvre rosse endolorie.

Hein, étés idiots,

Octobres malades,

Printemps, purges fades,

Hivers tout vieillots?

- «Quel silence, dans la forêt d'automne,

Quand le soleil en son sang s'abandonne! ( (Laforgues, 76)

À l'échelle du poème, dont nous ne citons qu'un extrait, l'oralité, les répétitions, la longueur variable des vers ainsi que la juxtaposition des groupes nominaux réduisent le langage à des voix, à des bruits. Les allitérations de sifflantes notamment évoquent la sensation lancinante de la rengaine sentimentale. La sensation de détresse et de mélancolie demeure mais le lecteur doit la reconstruire à partir d'éléments hétéroclites et contradictoires. Le sujet se dit en creux dans les perceptions plurielles qu'il génère chez le lecteur. Comme lorsqu'il recourt à la chanson, Laforgue cherche à valoriser la dimension sensuelle, concrète et performative du lyrisme pour arracher l'émotion à une littérarité sclérosante. Il libère la potentialité affective de la parole, en germe dans le texte. Sa poétique transforme l'écriture en « événement figural », tel que le définit Laurent Jenny dans la Parole singulière (Jenny, 15).

Enfin, la poétique synesthésique participe à la transgénéricité du lyrisme parce qu'elle bouleverse les termes de la communication. La réception multisensorielle diffracte la relation entre le sujet et le monde, entre le signe et la réalité, faisant surgir un autre référent, synthèse de plusieurs perceptions : d'où l'importance du principe kaléidoscopique, qui naît de la médiation plurisémiotique. C'est le cas de « Fleurs » de Rimbaud :

Laurence Tibi. La lyre désenchantée. L'instrument et la voix humaine dans la littérature française du XIXe siècle. Paris : Champion (Coll. « Romantisme et modernité »), 2003. 
D'un gradin d'or, - parmi les cordons de soie, les gazes grises, les velours verts et les disques de cristal qui noircissent comme du bronze au soleil, - je vois la digitale s'ouvrir sur un tapis de filigranes d'argent, d'yeux et de chevelures.

Des pièces d'or jaune semées sur l'agate, des piliers d'acajou supportant un dôme d'émeraudes, des bouquets de satin blanc et de fines verges de rubis entourent la rose d'eau.

Tels qu'un dieu aux énormes yeux bleus et aux formes de neige, la mer et le ciel attirent aux terrasses de marbre la foule des jeunes et fortes roses. (Rimbaud, 483)

Outre la superposition métaphorique du minéral et du végétal, d'objets naturels et culturels, du tactile et du visuel, on assiste à un véritable renversement de la perspective entre le sujet et le monde grâce à l'isotopie spectaculaire. La théâtralité du décor ne concerne pas seulement le « je » mais les végétaux qui l'entourent et qui deviennent également le foyer de la vision. À l'origine du regard, le « je » se dissout dans la pluralité du monde et de ses représentations. Les sensations se démultiplient et les émotions circulent entre les différents pôles de la communication.

Au terme de ce bref parcours, on peut dire que la synesthésie participe au renouvellement du lyrisme dans la mesure où elle bouleverse les modalités d'expression et de réception des émotions. Les poètes luttent ainsi contre des représentations conceptuelles et perceptuelles figées par la tradition artistique. Dès lors, l'écriture multisensorielle inscrit la transmission affective dans un mouvement fluctuant, multipolaire et intersubjectif que doit actualiser le lecteur. Transgénérique, le lyrisme synesthésique déplace l'expression des sentiments dans l'intervalle entre le sujet et l'objet, la création et la réception, l'intelligible et le sensible. 


\section{Ouvrages Cités}

BAUDELAIRE Charles. Les Fleurs du Mal (1857), Euvres complètes. Paris : Laffont (Coll. « Bouquins »), 1980. BERGEZ Daniel. Littérature et peinture. Paris : A. Colin, 2004.

BONNEFOY Yves. Lieux et destins de l'image. Un cours de poétique au Collège de France. Paris : Seuil, 1999. CORBIÈRE Tristan, Les Amours jaunes (1873). Paris : Seuil, 1992.

GHIL René, Traité du verbe, Paris : Giraud, 1886.

HAMBURGER Käte, Die Logik der Dichtung (1957). Logique des genres littéraires, traduit de l'allemand par Pierre Cadiot. Paris : Seuil, 1986.

JANKÉLÉVITCH Vladimir, La Musique et l'ineffable. Paris : A. Colin, 1961.

JENNY Laurent, La Parole singulière, préface de Jean Starobinski. Paris : Belin (Coll. « Poche »), 2009.

HOWES David. «L'esprit multisensoriel ». Communications, 86, 2010. Langages des sens [Numéro dirigé par Marie-Luce Gélard et Olivier Sirost] pp. 37-46. http://web/revues/home/prescript/article/comm_05888018_2010_num_86_1_2534

LAFORGUE Jules. Les Complaintes (1885). Pierre Reboul, ed. Paris : Lettres Françaises (Coll. de l'Imprimerie Nationale). 1981.

LAMBERT Hervé-Pierre. « La synesthésie. Vues de l'intérieur », Épistémocritique, Vol. VIII, Printemps 2011 (ISSN 1913-536X). http://www.epistemocritique.org/spip.php?article210.

LESSING Gotthold Ephraim. Laocoon ou Des limites respectives de la poésie et de la peinture (1766-1768). Paris : Hermann, 1990.

MALLARMÉ Stéphane. Euvres complètes. Paris : Gallimard (Coll. « La Pléiade »), 1945.

MONCOND'HUY Dominique, SCEPI Henri, eds. Les genres de travers. Littérature et transgénéricité. Rennes : Presses Universitaires de Rennes, 2007.

RIMBAUD Arthur. Euvres complètes. Pierre Brunel, ed. Paris : Livre de Poche (Coll. « La Pochothèque »), 1999. TIBI Laurence. La Lyre désenchantée. L'instrument et la voix humaine dans la littérature française du XIXe siècle. Paris : Champion (Coll. « Romantisme et modernité »), 2003.

VERLAINE Paul. Euvres poétiques complètes, Jacques Borel, ed. Paris : Gallimard (Coll. « La Pléiade »), 1962.

VOUILLOUX Bernard. La Peinture dans le texte. XVIIIe-XXe siècles. Paris : CNRS Éditions (Coll. « CNRS Langages »), 1995 (réimp. 1997). 